Derecho \& Realidad

Núm. 24 • II semestre de 2014

Facultad de Derecho y Ciencias Sociales, UPTC

ISSN: 1692-3936

\title{
El principio de la fraternidad en el correlato de los derechos humanos y el Estado social de derecho: hacia la construcción del estado de la cuestión del principio de la fraternidad en el derecho
}

The principle of fraternity in the correlate of the human rights and the social State of law: towards the construction of the state of the art of the principle of fraternity in the law

\section{Lizeth Judith Chocontá Solano*}

\section{Resumen}

En el relato constitucional se pretende seguir el rastro al principio de la fraternidad en la solución a las tensiones desatadas entre el Estado liberal, el modelo democrático radical y el socialismo moderno, presentadas a lo largo del siglo XIX y principios del siglo XX y cuya fórmula de equilibrio fue encontrada a través del Estado social de derecho. En el relato, los derechos humanos en perspectiva crítica, se pretenden establecer los puntos de encuentro y desencuentro entre la fraternidad y la tesis según la cual, los derechos humanos son el resultado de luchas de los pueblos, esto es, son creaciones colectivas plurales, diversas, con contextos sociales e históricos específicos.

\footnotetext{
* Abogada egresada de la Universidad Pedagógica y Tecnológica de Colombia, integrante del grupo de investigación Red-Humana de la Facultad de Derecho de la misma Universidad. Correo electrónico: lizju999@hotmail.com
} 
En este contexto es pertinente hablar de una teoría crítica, porque la complejidad de los derechos humanos exige interpretarlos y observarlos desde una visión relacional, holística y heterogénea, que incorpore una visión íntegra del ser humano. Se dice sobre el particular, que el método para su estudio debe mostrar los derechos humanos ante categorías sociales compuestas por: «ideas, instituciones, fuerzas productivas y relaciones sociales de producción, de género y étnicas, pero a la vez en su carácter impuro que tiene que ver con su posición, disposición, temporalidad-historicidad y narración. En su profundidad es preciso abordar su núcleo de concepción en el que aparecen espacios y valores, el desarrollo de las sociedades y las prácticas sociales». Con todo esto se pretende establecer un primer mapa que defina el estado del tema del principio de la fraternidad en el derecho constitucional.

\title{
Palabras clave
}

Fraternidad, Estado social de derecho, teoría crítica de los derechos humanos.

\begin{abstract}
In the constitutional narrative is intended to trace the beginning of the fraternity in the solution to the unleashed tensions between the liberal state, the radical democratic model and modern socialism, presented throughout the nineteenth and early twentieth century and which balance formula was found through the social rule of law. In the narrative of human rights in critical perspective, is intended to establish points of contact and divergence between fraternity and the thesis according to which human rights are the result of struggles of the people, i.e., they are plural collective creations, diverse, with specific social and historical contexts. In this context it is appropriate to speak of a critical theory because the complexity of human rights requires to interpret and to observe them from a relational, holistic and heterogeneous vision that incorporates an integration of human vision. It is said on the particular, that the method for study should show human rights in social groups composed of «ideas, institutions, productive forces and social relations of production, of gender and ethnic, but also in their impure nature having to do with their position, layout, temporality, historicity and narration. In their depth must address their core concept in which there are gaps and values, the development of societies and social practices». All this aims to establish an initial map that defines the state of the art of the principle of fraternity in constitutional law.
\end{abstract}

\section{Keywords}

Fraternity, social State of law, critical theory of human rights. 


\section{El constitucionalismo liberal y el origen de una tensión por falta del principio de la fraternidad}

Para abordar la temática propuesta es necesario tener presente el punto de inflexión generado por las revoluciones francesa y estadunidense de finales del siglo XVIII, así como los sucesos coyunturales que se presentaron en Inglaterra durante el siglo XVII. Estos permitieron definir y dar forma a las características del constitucionalismo moderno, el cual, bajo una fundamentación filosófico-política liberal, posibilitó el desarrollo de documentos jurídicos con modelos constitucionales novedosos e introdujo una nueva forma de legitimación, control y limitación del poder, así como demarcaciones y descripciones detalladas de la estructura y funcionamiento del Estado y catálogos de derechos bajo esquemas de una constitución escrita, particularmente en el caso francés y americano (Matteucci, 1998, p. 285).

Igualmente es preciso tener en cuenta que en el devenir de la Revolución francesa surge el muy conocido lema "libertad, igualdad y fraternidad", que implicó -en los términos de Antonio Baggio- arrancar a la fraternidad de las variadas interpretaciones de la tradición para insertarla en un contexto completamente nuevo, "junto a la libertad y a la igualdad, como tres principios ideales constitutivos de una perspectiva política inédita", pero con desenlaces disimiles, donde igualdad y libertad lograron una "evolución que las ha llevado a convertirse en verdaderas categorías políticas, capaces de expresarse no sólo como principios constitucionales, sino también como ideas-guía de movimientos políticos" (Baggio, 2006, p. 26); mientras que la fraternidad, como principio político, ha mantenido "una existencia marginal" con un recorrido de "río subterráneo".

Ahora bien, en este contexto y en un ejercicio de rastreo o al menos interpretación desde la fraternidad, es posible hacer una lectura de las tensiones que se originaron en el constitucionalismo liberal y que buscaron ser salvadas a través de la solución del Estado social de derecho:

Sumado a las evidencias advertidas por Antonio María Baggio frente a la idea de fraternidad en la Revolución francesa, se puede advertir en la idea de patria y nación, parte del fundamento de la fraternidad durante la Revolución francesa, pues el concepto de patria -como fuente de fraternidad- sería concebido como figura afectiva que implica vínculos de parentesco y amistad; no en vano, los diputados de París proclamaron el 5 de junio de 1790 "Franceses: ¡Somos hermanos! ¡Somos libres! ¡Tenemos una Patria!”

La explicación que se da al respecto en la obra titulada Pensar la solidaridad, es que la fraternidad que se invoca, en particular en el periodo jacobino, es una fraternidad política, "fundada en la pertenencia a una colectividad de libres e iguales. 
Por tanto, gracias a la mediación patriótica, la idea de fraternidad pudo unirse a las de libertad y la igualdad" (Villar \& García-Baró, 2004, p. 196).

Se continúa argumentando que la idea de fraternidad, en un primer momento, no remitirá a una simple virtud, un deber, o un sentimiento. Por el contrario, la afirmación revolucionaria de la idea de fraternidad se refiere a una situación concreta:

Somos hermanos, porque habiendo conquistado la libertad y la igualdad, al tiempo, hemos adquirido una patria. Por tanto, se trata no de una fraternidad religiosa o filosófica, sino de una fraternidad política, fundada en la pertenencia a una misma colectividad de libres e iguales. La plenitud de la igualdad es camaradería, saberse unidos en la búsqueda de un objetivo común (Villar \& García-Baró, 2004, p. 196).

$\mathrm{Al}$ respecto, Bloch propone su interpretación frente a la idea de fraternidad en la Revolución: "La lucha por la libertad crea la igualdad, la igualdad como final de la dependencia, mantiene la libertad, y la fraternidad es una recompensa de una igualdad en la que nadie necesita, ni tampoco pueden ser un lobo para los demás" (Bloch, 1980, p. 171).

Vale la pena hacer alusión a los comentarios hechos por Carlos de Cabo Martin, quien en clave de solidaridad ubica también en el primer constitucionalismo europeo o constitucionalismo del revolucionarismo francés, "el ideal laico de la fraternidad", "expresado en forma muy próxima a presupuestos básicos kantianos"; esto es, que el derecho positivo no se justifica por el cumplimiento de fines concretos, sino que tiene una fundamentación a priori, metafísica, en cuanto su cumplimiento es condición necesaria para la realización y el ejercicio de la libertad (De Cabo, 2006).

El citado autor advierte estos presupuestos en formulaciones como la Constitución jacobina de 1793, “o en la elevación de la idea de DEBER a principio básico del orden constitucional y, en consecuencia, como elemento de referencia colectiva, de 'todos' (art. 4 de la Constitución del año III), en cuanto solo partiendo de ese 'deber de todos' es posible la existencia de cada uno, es decir, de cada uno como sujeto de derechos" (De Cabo, 2006, p. 38).

Una lectura similar es hecha por el profesor Piero Costa, pero abordando el problema desde el análisis de los derechos. En efecto, el autor en mención advierte que los hoy conocidos derechos civiles y políticos, no son las únicas categorías de derechos que emergen en los debates revolucionarios franceses. Costa señala a Sieyès como el personaje que habló de un "derecho de socorro" que el ciudadano necesitado tiene frente a la nación; así como a Mart y Robesierre, quienes insistieron con 
mayor énfasis en el vínculo biunívoco que une a los sujetos con la nación: "de la misma manera que el ciudadano debe estar dispuesto a sacrificar sus bienes y su propia vida para salvar la república, la nación debe hacerse cargo de las necesidades fundamentales de sus miembros menos 'afortunados'. La Constitución jacobina de 1793 da una formulación precisa de este asunto hablando del 'deber sagrado' que la nación tiene para con los ciudadanos necesitados" (Costa, 2004, p. 54). No obstante, es importante aclarar que en la práctica, la pretensión jacobina establecida para superar las desigualdades sociales no llegó más allá de acciones de carácter eminentemente asistencialista.

En este punto el autor italiano Piero Costa pone de relieve el problema que es abierto para ser debatido a lo largo de los siglos XIX y XX, ¿basta con reconocer la existencia de un deber general de asistencia por parte del ente colectivo para atribuir a los sujetos el correspondiente e inviolable derecho? A lo que se responde inicialmente que "Lo importante es el hecho de que los sujetos adquieran, frente al ente colectivo, una expectativa -el 'derecho de socorro del que habla Sieyès- no reductible a la tutela de la libertad y de la propiedad y, en el peor de lo casos, inspirada por otros símbolos clave del ethos revolucionario como la igualdad y la fraternidad" (Costa, 2004, p. 55).

Esta respuesta rechaza de tajo la posibilidad de integrar, en términos jurídicos, la idea de fraternidad como principio vinculante. Este rechazo puede ser explicado, entre otras razones, porque a lo largo de los siglos XIX y XX, la idea de solidaridad irá desarrollándose y posicionándose frente al concepto de fraternidad; fundamentalmente porque la solidaridad, entendida como la dependencia mutua, permite ser incorporada en el ordenamiento jurídico, mientras que "la relación fraternal parece expresar sin más afectividad y subjetividad" (Villar \& GarcíaBaró, 2004, p. 197); esto es, "la relación fraternal es esencialmente una relación afectiva que excluye de su alianza a quienes no son miembros de ella" (Villar \& García-Baró, 2004, p. 197).

Reafirmando la convicción de que la fraternidad, a diferencia de la libertad e igualdad, no podría constituirse en derecho, entre otras razones, por su componente afectivo. Argumento que explica en alguna medida la razón por la cual el principio de fraternidad no está inserto dentro del constitucionalismo liberal; sin embargo es preciso indagar por otras razones de fondo que puedan explicar la evidente marginación de la fraternidad dentro del constitucionalismo liberal.

\subsection{Constitucionalismo liberal y la idea de fraternidad}

Dentro de la literatura especializada dedicada al estudio de la teoría constitucional, en especial del constitucionalismo liberal, es casi nula la presencia de análisis y 
acotaciones sobre el principio de la fraternidad, razón por la cual se debe indagar sobre la idea de fraternidad a través de los escritos hechos en clave de solidaridad -advirtiendo naturalmente, la clara diferencia entre una y otra ${ }^{1}$. De esta forma se pretende identificar el camino del "río subterráneo" que ciertamente ha afrontado la fraternidad.

En este intento se acudirá a las acotaciones sobre la inexistencia de solidaridad en el constitucionalismo liberal hechas en la obra titulada Teoría constitucional de la solidaridad. La tesis expresada al respecto es no solamente que el constitucionalismo liberal guarda silencio sobre la solidaridad o que la solidaridad no existe dentro del sistema constitucional liberal, sino que la "insolidaridad" es "un principio constitucional" necesario en el sistema liberal: "Precisamente porque es el constitucionalismo formal, del universalismo, de la abstracción, del sujeto (individual), en definitiva y paradójicamente 'el constitucionalismo de la igualdad'" (De Cabo, 2006, p. 44).

Se argumenta en la obra citada, que el esquema de las constituciones liberales establece un triple garantismo: en el ámbito privado (el de la extracción de la plusvalía) con la garantía de la libertad e igualdad; en el ámbito público a través de la división del poder y la garantía de coexistencia mediante la congruencia o correspondencia entre estos dos ámbitos necesarios en el modo de producción capitalista. Añade que el sistema constitucional completa la realización del individualismo, produciendo "un desconocimiento constitucional de clase", aunque la constitución sí realiza otra operación de clase: "la vertebración que a su través conduce a la unidad del Estado reconstruye y homogeniza a ese nivel el interés general"; pero con una peculiaridad: el interés general es ahora el interés general dominante.

El resultado de este proceso de configuración de la subjetividad individual se construye negando su existencia real en el orden social, de manera que el individuo real se disuelve en el orden jurídico (Díaz \& Olivas, 1997), “y por tanto, la abstracción y formalización que implica conducen no sólo a ocultar la desigualdad y la diferencia, sino a impedir que la desigualdad y la diferencia -o lo que es lo mismo, el conflicto- pase al orden jurídico" (Díaz \& Olivas, 1997, p. 43).

Se concluye en esta interpretación del constitucionalismo liberal, que a partir de la configuración del sujeto, se crean las bases de un sistema estructuralmente insolidario, en cuanto el sujeto lo es en sí y para sí exclusivamente,

Según Baggio (2006), la fraternidad exige una relación horizontal, mientras que la solidaridad implica una relación vertical donde no hay reconocimiento del otro. 
un absoluto en sí mismo y cuyo mecanismo central de participación, mediación y realización es en y a través del mercado. En esas condiciones (y en cuanto el otro, no existe, sino como posible competidor o potencial enemigo), la desintegración social en sentido literal es el resultado, en cuanto quedan excluidos tanto los que no pueden participar; mediarse o relacionarse a través del mercado (Díaz \& Olivas, 1997, p. 44).

Esta interpretación parece estar en consonancia con la crítica marxista al constitucionalismo liberal, entre otras razones, porque el formalismo que lo identifica impide observar el carácter unitario del ser humano en sociedad, estableciendo una falacia formalista y universalista que descuida el problema de la desigualdad material de la que son víctimas las clases trabajadoras.

Si se apareja esta interpretación estudiada a la luz de la fraternidad en clave de solidaridad, es posible cuestionar la premisa según la cual, la fraternidad, a diferencia de la libertad e igualdad, no podría constituirse en derecho o principio jurídicopolítico, por su componente afectivo.

La razón fundamental es precisamente que el intento de abstracción jurídica de un sujeto de derecho en el marco de una igualdad formal, opacó la realidad material de los ciudadanos en sus relaciones personales, sus desigualdades materiales y sus diferencias.

Sumado a lo antes dicho, la estructura del constitucionalismo liberal trajo consigo desencuentros intrínsecos que originaron tensiones ampliamente discutidas y cuestionadas en los años posteriores.

\subsection{La tensión entre liberalismo y democracia en el constitucionalismo liberal}

El sustrato racionalista e iluminista que acompañó la Revolución francesa de 1789, permitió la aparición de una libertad inédita, que se apartó de aquella visión del constitucionalismo histórico inglés que daba a la libertad una concepción historicista. En efecto, la constitución concebida como garantía de libertades necesariamente requería ser obra de la razón: "De ahí que la libertad a la francesa sea una libertad nueva y abstracta que hay que constituir según un modelo ideal, mientras que la libertad a la inglesa sea una práctica antigua que hay que conservar y perfeccionar" (Guerra, 2010, p. 30).

Igualmente, las declaraciones de derechos como la de 1789, tuvieron fundamento iusnaturalista, esto es, los derechos fundamentales no se crean sino que se declaran, en tanto que los derechos existen como acervo inescindible de la naturaleza humana. Así mismo se confeccionó un catálogo de derechos donde se consignaron derechos 
de libertad, propiedad y resistencia, todos ellos de manifiesta importancia doctrinal desarrollados y debatidos durante el denominado siglo de las luces. También aparece un nuevo elemento legitimador del nuevo orden estatal a partir del concepto de soberanía nacional, que anuncia a la nación como poder constituyente y nuevo soberano.

Todos estos elementos integraron las características del constitucionalismo moderno, que, desde sus inicios, implicó el desarrollo de constantes tensiones que trataron de ser salvadas muchas décadas después. En efecto, el carácter liberal del constitucionalismo moderno implicó contraponer "el liberalismo, que privilegia la calidad y la diferencia, a la democracia, que es el dominio de la calidad y el reino de la uniformidad" (Matteucci, 1998, p. 272). Por esta razón, a pesar de que las distintas declaraciones establecían el derecho al voto, proclamaban la soberanía nacional y hablaban de igualdad, en la práctica no se aplicaba la democracia directa y universal; en otras palabras, el sistema representativo del constitucionalismo moderno estableció grandes restricciones a los derechos de participación política, que se tradujeron en un régimen poco democrático.

En igual sentido y a pesar de que los catálogos de derechos (civiles y políticos), y el concepto de soberanía nacional representa -en los términos de Piero Costa- los ejes del nuevo orden, no son elementos pacíficamente complementarios y por el contrario, constituyen otro campo de tensión:

El carácter natural, no voluntario, no negociable de los derechos no solo encuentra un difícil composición potestativa y voluntarista de la soberanía nacional y de la ley; la libertad privada, la libertad-propiedad, entra en tensión con otra componente, también esencial, de la libertad: la libertad política, la libertad-participación (Costa, 2004, p. 54).

\section{El Estado social de derecho y el principio de la fraternidad}

La fórmula del Estado social de derecho es considerada un resultado complejo, a veces contradictorio, como consecuencia de debates políticos, filosóficos, sociológicos, económicos y jurídicos desarrollados con bastantes años de precedencia, pero que evidentemente tienen como punto de partida y principal antecedente, el constitucionalismo liberal. Como el objetivo de este artículo está enfocado en la elaboración del estado del tema de la fraternidad, bien como principio ${ }^{2}$,

\footnotetext{
Principios constitucionales: consagran prescripciones jurídicas generales que suponen una delimitación política y axiológica reconocida y, en consecuencia, restringen el espacio de reinterpretación, lo cual hace de ellos normas de aplicación inmediata, tanto por el legislador como por el juez constitucional. (Corte Constitucional Colombiana, sentencia C-1287 de 2001, MP Marco Gerardo Monroy Cabra).
} 
bien como regla ${ }^{3}$ o bien como valor ${ }^{4}$ jurídico, no se abordarán las variables y presupuestos históricos que rodearon la formulación del Estado social de derecho, apenas se harán algunos apuntes que nos facilitan el análisis de nuestro tema de estudio: la idea de fraternidad en el derecho constitucional y el Estado social de derecho.

Como se ha subrayado, el Estado social de derecho corresponde a una fórmula que amalgama elementos que en determinados momentos fueron contradictorios:

De un lado, es un Estado liberal, que recoge las aspiraciones del Estado de derecho clásico del Siglo XIX. Según esta filosofía, lo importante es el control al Estado, a fin de limitarlo por mecanismos como la división de poderes y el control constitucional (Uprimny, 1995).

De otro lado, es un Estado democrático, que reposa en la soberanía popular, en la formación de la voluntad política. Lo importante desde esta perspectiva es el origen de las decisiones jurídicas y políticas, por lo cual la democracia, conforme a las clásicas enseñanzas de Rousseau, es entendida como un procedimiento igualitario de formación de poder con base en el predominio del principio de la mayoría (Uprimny, 1995).

Finalmente es un Estado social, que reposa en la idea de que el poder político debe lograr para los asociados una justicia material mínima: lo importante es entonces el resultado de la acción pública, de suerte que la democracia es entendida más como un efecto material del ejercicio del poder, esto es, como cierta igualdad social conseguida gracias a la acción estatal. Esta concepción está asociada entonces a la llamada libertad positiva, es decir, aquella que deriva de la existencia de prestaciones positivas por parte del Estado (Uprimny, 1995).

Ahora bien, teniendo presente la conformación del Estado social de derecho, se puede observar que al contrario del constitucionalismo liberal, que era el constitucionalismo de la igualdad, el Estado social de derecho admite la posibilidad de la diferencia. Esta posibilidad abre la puerta para que la fraternidad como principio jurídico pueda tener espacio dentro de un ordenamiento jurídico, así sea actuando

3 Reglas constitucionales: son disposiciones jurídicas en las que se define en forma general y abstracta un supuesto de hecho y ordenan una consecuencia jurídica. (Corte Constitucional Colombiana, sentencia C-1287 de 2001, MP Marco Gerardo Monroy Cabra).

4 Valor constitucional: los valores representan el catálogo axiológico a partir del cual se deriva el sentido y la finalidad de las demás normas del ordenamiento jurídico. Independientemente de su consagración explícita o no, estos constituyen el fundamento y la finalidad de la organización política. (Corte Constitucional Colombiana, sentencia C-1287 de 2001, MP Marco Gerardo Monroy Cabra). 
como un "río oculto" que actúa marginalmente como un elemento corrector del sistema.

La particularidad descrita anteriormente puede ser corroborada en el ámbito del derecho constitucional contemporáneo, cuando elaboró una visión integral y realista del ser humano para superar el concepto individualista que rigió la filosofía política del siglo XIX. Pues entendió -en los términos de la sentencia T-659 de 2007 de la Corte Constitucional colombiana-, que la plena protección de los derechos no solamente abarca la dimensión subjetiva y particular del hombre, sino que también debe dirigirse a su dimensión social, la cual supone una concepción del ser humano en sociedad y en relación con el otro.

Así, la positivización de los derechos colectivos, para referirse a aquellos que desbordan el interés puramente individual, y de los derechos fundamentales, como aquellos que se materializan de manera subjetiva, simplemente refleja esa nueva visión del derecho que si bien es cierto considera al individuo como fundamento y fin último del Estado, no lo es menos que su protección no se limita a la abstención o prestación subjetiva, sino que incluye en el marco de acción pública ese contexto en el que el hombre hace parte de un interés común, cuya titularidad compartida de sus derechos pretende un destino común. (Corte Constitucional, sentencia T-659 de 2007)

Con el nuevo panorama del derecho constitucional se puede advertir el colapso de la tesis que consideró a los derechos fundamentales y colectivos como dos formas excluyentes de protección de los intereses del ser humano. La señalada providencia T-659 de 2007 de la Corte Constitucional colombiana, explica que este fenómeno se presentó cuando el aplicador jurídico se enfrentó a casos en los que un derecho, que había sido considerado colectivo, se convertía en un derecho individual al afectar el núcleo esencial de un derecho subjetivo (piénsese en el caso de los pescadores que no pueden ejercer su oficio cuando hay un grave daño ambiental). $\mathrm{O}$, incluso, se presentan situaciones en las que un derecho típicamente fundamental se torna en derecho de una colectividad que se beneficia en forma común al individuo afectado (por ejemplo, el caso del derecho a la libertad de locomoción que se encuentra afectado por ausencia de medios de transporte).

Esta posición, indicada a manera de ejemplo por la Corte Constitucional colombiana, manifiesta la presencia y actuación de la idea de la fraternidad a través de una de sus características: el reconocimiento de la dimensión social del hombre a la hora de tutelar un derecho subjetivo fundamental, y el reconocimiento del hombre en relación, es decir, el reconocimiento de la realidad del otro. Sin embargo, es necesaria una fundamentación jurídico-teórica que visibilice la acción de la 
fraternidad dentro de las relaciones sociales del ser humano, tarea que evidentemente está sin iniciar; pues el principio de la fraternidad no puede estar marginado al desarrollo de un ejercicio corrector de las desigualdades e injusticias generadas por el sistema capitalista. Se hace necesario integrar la fraternidad como principio activo de los ordenamientos jurídicos, a fin de atender los reales requerimientos y necesidades del ser humano en toda su extensión.

\section{La idea de fraternidad y la teoría crítica de los derechos humanos}

Para finalizar la propuesta, es necesario hacer unas breves acotaciones frente a los derechos humanos en perspectiva crítica, para establecer los puntos de encuentro y desencuentro entre fraternidad y la tesis según la cual, los derechos humanos son el resultado de luchas de los pueblos, esto es, son creaciones colectivas plurales, diversas, con contextos sociales e históricos específicos. En este contexto es pertinente hablar de una teoría crítica, porque la complejidad de los derechos humanos exige interpretarlos y observarlos desde una visión relacional, holística y heterogénea, que incorpore una visión íntegra del ser humano. Se dice sobre el particular que el método para su estudio debe mostrar los derechos humanos ante categorías sociales compuestas por: "ideas, instituciones, fuerzas productivas y relaciones sociales de producción, de género y étnicas, pero a la vez en su carácter impuro que tiene que ver con su posición, disposición, temporalidad-historicidad y narración. En su profundidad es preciso abordar su núcleo de concepción en el que aparecen espacios y valores, el desarrollo de las sociedades y las prácticas sociales". Con todo esto se pretende establecer un primer mapa que defina el estado del arte del principio de la fraternidad en el derecho constitucional.

Se sostiene que la perspectiva crítica avanza hacia el encuentro de respuestas más completas que las dadas por el derecho y el mismo constitucionalismo liberal, para lo cual parte de una dimensión política social, ética y jurídica. En la dimensión ética se expresa la dignidad, en cuanto vivir implica construir las bases materiales y mentales del sujeto. "En los contenidos de los derechos no hay un único sentido de dignidad, no existe una dignidad universal, sino múltiples dignidades asociadas a las raíces profundas de cada cultura en cuanto los pueblos viven, sienten desean, de maneras distintas, que se conectan en el sentido humanizante" (Restrepo, 2010).

La dimensión política de los derechos humanos proviene del campo de la justicia; "en ella radican la igualdad y la libertad, que no deben ir separadas; son mellizas, cada una es un pilar del discurso y de los derechos humanos. La libertad se funda en la necesidad del ser humano por descubrir el tiempo y el espacio. La igualdad se reconoce a través de las capacidades y oportunidades para alcanzar las delicias de hablar en el tiempo y el espacio." Al respecto, manifiesta el profesor Manuel 
Humberto Restrepo que la libertad -para la perspectiva crítica- no pone al otro como límite, sino como oportunidad para extender las libertades. Esta libertad e igualdad en el plano colectivo tejen la solidaridad.

En cuanto a la solidaridad, el autor manifiesta que esta es la tercera dimensión de los derechos humanos, porque da el carácter social de los derechos, de producto cultural colectivo, creado por la lucha de los pueblos en cuanto los evocan y los convierten en símbolos. Finalmente aparece la dimensión jurídica, la cual regula y positiviza en normas comunes los derechos resultados de las luchas sociales; sin embargo, el autor advierte que el derecho en este punto puede ser peligroso, porque puede individualizar y subjetivizar lo que en esencia es colectivo.

Estas dimensiones de la teoría crítica de los derechos humanos permiten advertir semejanzas respecto a la idea de fraternidad; no obstante se aleja del concepto prefiriendo hablar en clave de solidaridad. Igualmente parte de una inversión de principios que históricamente está probada que no tiene efectos positivos. No es de la libertad y la igualdad que se llega a la solidaridad y a la acción social. Por el contrario, es a partir del ejercicio del reconocimiento de la realidad del otro en el plano de la relación social corresponsable, de donde se parte para llegar a la libertad e igualdad del ser humano.

Como se evidenció a lo largo del trabajo presentado, la fraternidad ha sido marginada del debate político, filosófico y naturalmente jurídico, sin embargo, como se pudo demostrar, siempre ha estado presente, manifestada en sus elementos característicos: la dimensión social del hombre y el reconocimiento de la realidad del otro.

\section{Referencias}

Baggio, A. M. (2006). Introducción al principio olvidado. El redescubrimiento de la fraternidad en la época del Tercer '89'. Buenos Aires: Ciudad Nueva.

Bloch, E. (1980). Derecho natural y dignidad humana. Madrid: Aguilar.

Corte Constitucional Colombiana (2001). Sentencia C-1287. M.P. Marco Gerardo Monroy Cabra.

Costa, P. (2004). 'Derechos' en el Estado moderno en Europa. Madrid: Trotta.

De Cabo, C. (2006). Teoría constitucional de la solidaridad. Madrid: Marcial Pons, Ediciones Jurídicas y Sociales. 
Díaz, O. \& Olivas, E. (1997). Metafísica e historicidad de los derechos. Madrid: Dykinson.

Guerra, X. (2010). Modernidad e independencias, ensayos sobre las revoluciones hispánicas. Madrid: Fondo de Cultura Económica.

Matteucci, Nicola. (1998). Organización del poder y libertad: historia del constitucionalismo moderno. Madrid: Trotta, Universidad Carlos III de Madrid, Departamento de Derecho Público y Filosofía del Derecho.

Restrepo, M. H. (2010). Teoría crítica de los derechos humanos. Tunja: Universidad Pedagógica y Tecnológica de Colombia.

Uprimny, R. (1995). La motivación de las sentencias y el papel del juez en el Estado social y democrático de derecho. En Pensamiento Jurídico, no. 4. Bogotá: UN.

Villar, A. \& García-Baró, M. (2004). Pensar la solidaridad. Madrid: Universidad Pontificia de Comillas. 